\title{
Apps para o Ensino de Matemática: Descritores como Metadados para Busca de um Repositório de Objetos de Aprendizagem
}

\author{
Elvis Medeiros de Melo ${ }^{1}$, Clésia Jordânia Nunes da Costa ${ }^{2}$ \\ ${ }^{1}$ Instituto Metrópole Digital - Universidade Federal do Rio Grande do Norte (UFRN) - Natal \\ - Brasil \\ ${ }^{2}$ Departamento de Matemática - UFRN - Natal - Brasil \\ \{elvismedeiros.mm, clesiaj7\}@gmail.com
}

\begin{abstract}
This article de, als with the continuation of the research work developed in the Objets of Learning for Mathematics (OBAMA) research group that aims to catalog and classify Learning Objects (OA) for Mathematics, with the aim of providing access to $O A$ to help integrate technology into the classroom. In this paper, we will cover the classification of the 201 apps as Mobile Learning Objects (OAM) for mathematics, aiming at the skills defined by the Curricular Common National Base (BNCC). The OAM were classified in skills directed to each stage of both the earliest years and the final years. Among the results, 42 apps that did not apply to any skill and 37 apps were discontinued.
\end{abstract}

Resumo. O presente artigo trata da continuação do trabalho de pesquisa desenvolvido no grupo de pesquisa Objetos de Aprendizagem para Matemática (OBAMA) que tem por objetivo catalogar e classificar Objetos de Aprendizagem (OA) para Matemática, com o intuito de oportunizar o acesso a OAs para auxiliar na integração da tecnologia na sala de aula. Neste trabalho, abordaremos a classificação dos 201 aplicativos (apps) considerados como Objetos de Aprendizagem Móveis (OAM) para matemática visando as habilidades definidas pela Base Nacional Comum Curricular. Os OAM foram classificados em habilidades voltadas para cada etapa de ensino, tanto os anos iniciais quanto os anos finais do ensino fundamental. Dentre os resultados, 42 apps que não se aplicam em alguma habilidade e 37 apps foram descontinuados.

\section{Introdução}

As ferramentas para auxiliar no ensino de matemática sempre fizeram parte dos avanços tecnológicos de cada época, desde materiais concretos usados para fazer a relação na contagem de objetos ao uso de um celular para se aplicar, revisar e pesquisar um conceito matemático, assim afirma Wiley (2008) ao dizer que Objeto de Aprendizagem (OA) é qualquer material que possa ser reutilizável, assim como também afirma Batista et al (2017, p. 60), “[...] os OA possuem ainda, características didático-pedagógica, pois estão direcionados a uma parte ou um conjunto de partes de determinado conteúdo". Podemos inferir assim que os OA podem ser usados na sala de aula como uma ferramenta que pode auxiliar ao professor na sua prática docente.

Com a proposta de enriquecer os processos de ensino e de aprendizagem, no contexto dos aplicativos (apps) e o saber ao alcance de todos viabilizado pela web, trazemos o nosso trabalho que tem o objetivo de apresentar o processo de catalogação e classificação de apps educativos para dispositivos móveis incluídos na Plataforma Objetos de Aprendizagem para 
Matemática (OBAMA) ${ }^{1}$, classificados em níveis de ensino da Educação Básica e em descritores de habilidades da Base Nacional Comum Curricular [BNCC 2017]. Para a Matemática, os descritores da BNCC se distribuem nas seguintes unidades temáticas: Números; Álgebra; Grandezas e Medidas; Geometria e Estatística e Probabilidade. Essa pesquisa tem a finalidade de oportunizar aos professores da educação básica o acesso a recursos que auxiliem na integração das Tecnologias Digitais de Informação e Comunicação (TDIC) com a aprendizagem matemática.

Em se falando de aprendizagem matemática em um contexto regional, segundo dados da Plataforma QEdu, que baseia suas pesquisas e inferências em avaliações de larga escala aplicadas aos estudantes brasileiros de escolas públicas, apenas $20 \%$ dos alunos potiguares do $5^{\circ}$ ano do Ensino Fundamental possuem aprendizado matemático adequado. Esse índice é ainda pior para o $9^{\circ}$ ano, em que somente $8 \%$ dos discentes têm a proficiência matemática indicada para a faixa etária escolar [QEDU 2018]. Isso implica que a quase totalidade dos estudantes do estado chega à última fase do Ensino Fundamental com sérias dificuldades em Matemática.

Nesse contexto, entendemos que os Objetos de Aprendizagem Móveis (OAM) são OA para dispositivos móveis e podem ser usados como recursos educacionais no processo de ensino e aprendizagem. Propomos a classificação de 201 apps para matemática para os anos iniciais e finais da educação básica com base nas habilidades descritas pela BNCC, que define "[...] o conjunto orgânico e progressivo de aprendizagens essenciais que todos os alunos devem desenvolver ao longo das etapas e modalidades da Educação Básica" [BNCC, 2017, p. 7]. Nesse momento a BNCC está focada na Educação Infantil e o Ensino Fundamental, com a proposta para o Ensino Médio já em sua primeira versão publicada. Para o escopo da pesquisa, utilizamos apenas o documento final da BNCC, focando nossa análise para o Ensino Fundamental.

Este artigo se organiza em cinco seções. Além desta Introdução; Fundamentação Teórica, na qual discorremos acerca de trabalhos anteriores, o uso do celular na sala de aula e importância do uso de apps na educação matemática; Metodologia, na qual mostramos os passos do trabalho realizado; Resultados e discussões, onde analisamos os dados obtidos a partir do levantamento e classificação dos OAM; e finalizamos com as Considerações que o estudo proporcionou.

\section{Fundamentação Teórica}

Vivemos em Ciberespaço em que parte da consciência humana do século XXI é digitalizada [Lemos 2010]. Isto se deve, principalmente, à popularidade adquirida por dispositivos móveis como laptops, tablets e, especialmente, os smartphones. A proibição do uso do celular em espaços educacionais, proposta por alguns educadores, não se sustenta. Quando bem planejados e integrados em práticas educativas, tais dispositivos e seus recursos deixam de ser instrumentos que desviam a atenção discente, para tornarem-se aliados a práticas de ensino dos professores que coloquem os estudantes mais ativos, colaborativos e autônomos na construção do conhecimento.

Quando direcionamos o olhar para o ensino da Matemática identificamos problemas alarmantes. Segundo relatório publicado pela Organização para a Cooperação e Desenvolvimento Econômico (OCDE), o Brasil está entre os dez países com pior rendimento

\footnotetext{
${ }^{1}$ Disponível em: <Obama.imd.ufrn.br>. Acesso em: 29 set. 2018
} 
escolar em matemática, ciências e leitura [OCDE 2016]. Ainda segundo o estudo, $67,1 \%$ dos alunos brasileiros apresentam um baixo rendimento e proficiência em matemática.

Em 2016, a pesquisa TIC Educação confirma tendências importantes já verificadas ao longo da série histórica, e também inclui novos indicadores que passaram a ser monitorados. Pela primeira vez, a utilização de celulares em atividades escolares foi investigada entre os alunos. O uso desse tipo de dispositivo foi citado por $52 \%$ dos alunos de escolas com turmas de $5^{\circ}$ e $9^{\circ}$ anos do Ensino Fundamental e/ou $2^{\circ}$ ano do Ensino Médio, localizadas em áreas urbanas [CGI.BR 2017].

Apesar do avanço no uso do celular enquanto ferramenta pedagógica, o mesmo estudo aponta apenas que $31 \%$ dos estudantes afirmaram utilizar a Internet por esse tipo de equipamento na escola, sendo $30 \%$ entre os alunos de escolas públicas e $36 \%$ nas instituições privadas. As restrições ao acesso de estudantes à rede WiFi da escola estão entre os aspectos que explicam a baixa utilização desse dispositivo no ambiente escolar [CGI.BR 2017].

A motivação para esse descompasso, dentre outros aspectos, pode estar na infraestrutura necessária para essas práticas, em especial, conexão à internet banda larga e acesso aos dispositivos e apps [Melo et al 2017]. Nesse sentido, o desenvolvimento de repositório que oportunize aos professores acesso aos apps educativos, classificados de acordo com habilidades e competências matemáticas, pode facilitar a integração de tais TDIC nas aulas de Matemática.

Delimitar critérios de qualidade para a escolha de aplicativos implica em saber analisar de que forma essa tecnologia poderá ter um uso educacional, refletindo se a aprendizagem poderá ocorrer em um contexto de mobilidade e como vai possibilitar ao sujeito a construção de conhecimentos de formas individuais ou coletivas. Saccol, Schlemmer e Barbosa (2011), destacam que os aplicativos devem instigar as habilidades cognitivas dos alunos e, acima de tudo, proporcionar situações para que possam utilizar os novos conhecimentos para a solução de problemas.

Para o ensino e a aprendizagem da Matemática, Castro-Filho et al (2016) destacam que o acesso a diferentes fontes de informação, as múltiplas formas de representar o pensamento matemático e a manipulação dinâmica de símbolos matemáticos, sendo alguns dos elementos que favorecem o pensamento matemático apoiado por TDIC. Alguns apps oportunizam essas experiências. Além disso, a mobilidade e a conectividade proporcionada pelos apps podem possibilitar experiências pedagógicas relacionadas com novas formas de comunicar, registrar e representar o pensamento. O professor deve estar preparado para conhecer especificidades das TDIC para incluí-las em seu plano de aula e oportunizar práticas, efetivamente, inovadoras com seus alunos.

Em trabalho recente, Oliveira, Silva, Nunes e Maia (2017), trataram em seu trabalho de catalogação e classificação de OA para desktop, classificados em etapa de ensino e por tema curricular, de acordo com a matriz de referência da Prova Brasil. Na mesma perspectiva, Melo, Costa e Maia (2017) catalogam OAM para matemática, classificando em temas curriculares de acordo com a mesma matriz. Sobre os procedimentos metodológicos adotados na classificação e nova catalogação dos apps, discutimos a seguir.

\section{Metodologia}

Trata-se de um trabalho de cunho misto que envolve o quantitativo e o qualitativo dos OAM analisados, dispondo em tabelas e representando os resultados seus dados por meio de 
gráficos, assim como comentários acerca dos dados coletados e discussão. A classificação foi realizada com 201 apps para matemática anteriormente classificados temas curriculares da Prova Brasil e armazenados na Plataforma OBAMA. Na atual pesquisa, os apps foram classificados com base nos descritores de habilidades para cada ano do Ensino Fundamental e unidade temática da BNCC.

$\mathrm{Na}$ classificação dos OAM, usamos a validação em pares utilizando o conceito de usabilidade pedagógica de Muniz (2015). Ele sugere que o uso de sistemas digitais para educação deve ser avaliado levando-se em consideração questões específicas para o processo de ensino e de aprendizagem. Assim, a classificação dos OAM se deu nas seguintes etapas: (i) baixar os apps catalogados; (ii) testar cada app, jogando, explorando a interface para o nível de ensino adequado; e (iii) Analisar quais habilidades podem ser desenvolvidas por cada descritor da BNCC, assim como unidades temáticas. Com o descritor atribuído, conseguimos como metadado o ano de ensino adequado para o uso do app. Para cada etapa, um agente foi definido: Catalogador, Revisor e Validador. No outro momento, o mesmo objeto é testado por um segundo avaliador que valida ou não a classificação anterior, como ilustra a Figura 1.

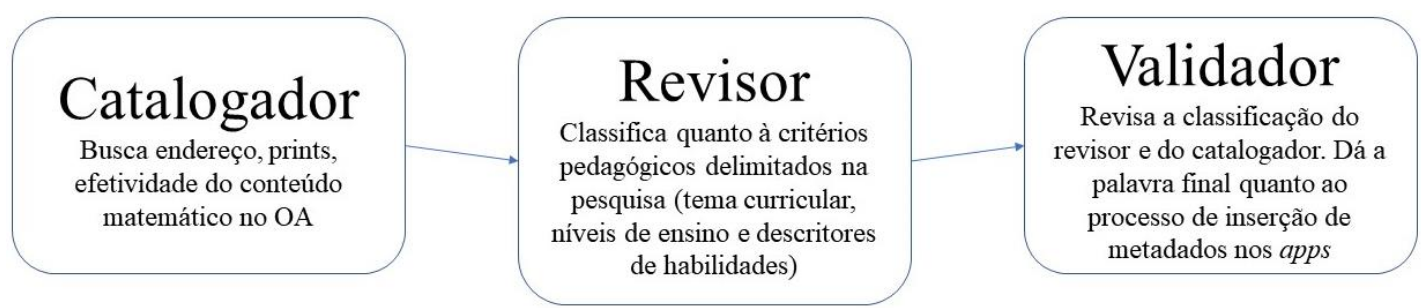

Figura 1. Esquema da Metodologia de classificação dos apps.

A proposta desse levantamento de apps é buscar os que possuem a permissão Creative Commons (CC) para que possamos disponibilizar o download na Plataforma OBAMA.

Para esse trabalho, foram usados os descritores de habilidades que vão $1^{\circ}$ ao $9^{\circ}$ ano do Ensino Fundamental. Para o $1^{\mathrm{o}}$ ano de Matemática, a BNCC propõe 22 habilidades, para o $2^{\circ}$ ano 23 habilidades, $3^{\circ}$ e $4^{\circ}$ ano 28 habilidades e $5^{\circ}$ ano tem 25 habilidades para os anos iniciais. Para os Anos Finais, o $6^{\circ}$ ano possui 34 habilidades, $7^{\circ}$ ano possui 37 , o $8^{\circ}$ ano possui 27 e o $9^{\circ}$ ano possui 23 . Essas habilidades são distribuídas em cinco unidades temáticas de Matemática, quais sejam: Números, Álgebra, Grandezas e Medidas, Geometria e Probabilidade e Estatística. Cada habilidade é identificada por um código que segue um protocolo que indica o Nível de Ensino (EF - Ensino Fundamental); o ano escolar (01 - $1^{\circ}$ ano); a disciplina (MA - Matemática) seguido de um número (01 a 22) que a específica, além do código de classificação das habilidades existentes. Cada descritor representa uma habilidade matemática ligada ao conteúdo estudado em sala de aula, proposto pelo bloco a ser explorado pelo professor, servindo como referência para que ele planeje e execute suas aulas com vistas ao desenvolvimento discente. Criamos uma novo código para representar os apps que "EF00MA00 - Não se aplicam" que, após processo de classificação, não se enquadraram em nenhum das habilidades propostas para o nível de Ensino Fundamental da BNCC.

Essa classificação foi realizada com base no uso de uma planilha eletrônica colaborativa. No processo de classificação em descritores de habilidades da BNCC, cada avaliador ficou responsável por preencher a planilha com as informações pedagógicas através da usabilidade desse recurso e adequação às habilidades propostas para o nível. Tal processo foi otimizado pela planilha desenvolvida (Ver Figura 2), haja vista trabalhos já realizados anteriormente com os recursos da Plataforma OBAMA. 
VII Congresso Brasileiro de Informática na Educação (CBIE 2018)

Anais dos Workshops do VII Congresso Brasileiro de Informática na Educação (WCBIE 2018)

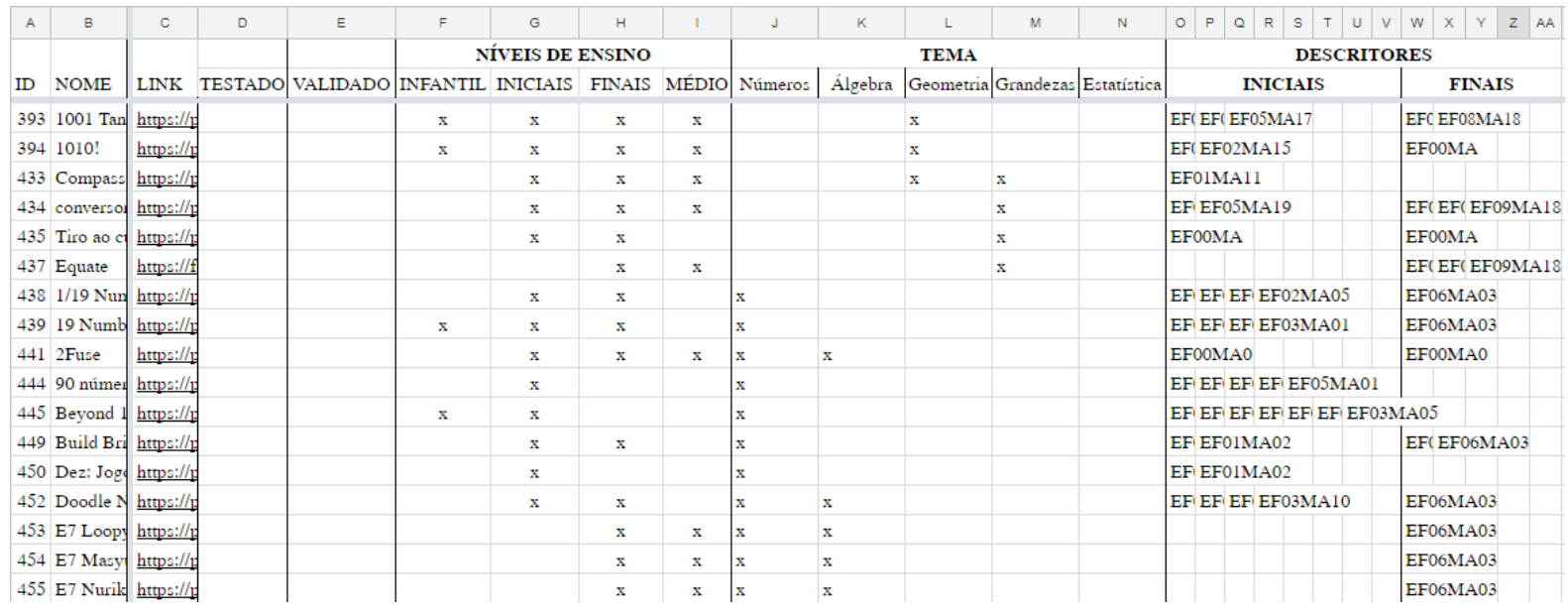

Figura 2. Planilha Eletrônica utilizada na classificação dos apps.

Nesse momento da pesquisa, realizamos a classificação dos OAM com base na funcionalidade e características dos apps de acordo com as habilidades, baixando os apps nos dispositivos móveis (tablet ou smartphone). Quando não era possível testar o $a p p$, fosse por limitação computacional ou incompatibilidade com o dispositivo móvel, utilizamos outras alternativas para testá-lo como: descrição do aplicativo na plataforma cadastrada; imagens das telas disponibilizadas pelo autor, assim como vídeos no YouTube que demonstram a interação do usuário com o app.

Ao consultar os apps anteriormente catalogados para a plataforma, percebemos que alguns foram descontinuados ou não estavam mais disponíveis para download. No processo de curadoria dos links, ou seja, a verificação dos links que estavam funcionando no processo de classificação em descritores da $\mathrm{BNCC}$, removemos um total de 37 apps. O quadro 1 mostra o quantitativo de apps catalogados por período, assim como os removidos após a curadoria.

\begin{tabular}{|c|c|c|}
\hline Período de Catalogação & $\begin{array}{c}\text { Quantidade de } \text { Apps } \\
\text { Catalogados }\end{array}$ & Removidos após curadoria \\
\hline Dezembro/2016 a Março/2017 & 187 & 17 \\
\hline Abril/2017 a Fevereiro/2018 & 61 & 0 \\
\hline Março/2018 a Agosto/2018 & 0 & 20 \\
\hline Total & 238 & 37 \\
\hline
\end{tabular}

Quadro 1. Quantitativo de apps por período de catalogação.

Considerando que a plataforma ainda possui algumas funções em implementação, para registro e análise neste artigo, selecionamos os OAM encontrados, catalogados e classificados até agosto de 2018. Atualmente, os recursos são apresentados por meio de uma busca, no qual o usuário pode escolher filtrar por níveis de ensino, bloco de conteúdos e descritores da Prova Brasil. Na plataforma ainda há recursos para desktop. A classificação detalhada para os recursos mobile, assim como definição da metodologia para classificação de OAM, ainda está em desenvolvimento. Dos 514 recursos catalogados e classificados na Plataforma OBAMA, destacamos 313 são para desktop e 201 são mobile.

\section{Resultados e Discussão}


VII Congresso Brasileiro de Informática na Educação (CBIE 2018)

Anais dos Workshops do VII Congresso Brasileiro de Informática na Educação (WCBIE 2018)

No processo de busca, a medida em que o usuário procura por apps similares, vê no perfil de desenvolvedores dos OAM, além da combinação de palavras-chave no buscador dessa ferramenta, o repositório de apps lhe fornece sugestões de outros recursos de acordo com as buscas frequentes.

Ao final da classificação foram analisados 201 apps, onde se encontram na Plataforma OBAMA, já classificados em níveis de ensino, unidades temáticas e descritores de habilidades da Prova Brasil. Neste trabalho, realizamos um recorte para a quantidade de dados relacionados ao número de objetos classificados por anos de ensino e as habilidades classificadas em cada um deles, relacionadas com a unidade temática para a BNCC. Na tabela 1 é apresentado o quantitativo de habilidades nos 201 apps classificados para cada ano de ensino dos anos iniciais e finais do Ensino Fundamental.

\begin{tabular}{|c|c|c|}
\hline Ano de Ensino & Habilidade & $\begin{array}{c}\text { Quantidade de Habilidades em } \\
\text { cada Ano }\end{array}$ \\
\hline $1^{\circ}$ & EF01M01 a EF01M22 & 207 \\
\hline $2^{\circ}$ & EF02M01 a EF02M23 & 140 \\
\hline $3^{\circ}$ & EF03M01 a EF03M28 & 189 \\
\hline $4^{\circ}$ & EF04M01 a EF04M28 & 114 \\
\hline $5^{\circ}$ & EF05M01 a EF05M25 & 79 \\
\hline $6^{\circ}$ & EF06M01 a EF06M34 & 174 \\
\hline $7^{\circ}$ & EF07M01 a EF07M37 & 125 \\
\hline $8^{\circ}$ & EF08M01 a EF08M27 & 95 \\
\hline $9^{\circ}$ & EF09M01 a EF09M23 & 37 \\
\hline
\end{tabular}

Tabela 1. Número de apps por ano de ensino por descritores de habilidade.

Com base nos dados apresentados, a medida em que os anos de ensino passam, o número de apps classificados em cada uma dessas etapas diminui. A relação de OAM voltados aos anos de fechamento de cada ciclo de cada nível de ensino, respectivamente o $5^{\circ}$ ano dos anos iniciais e $9^{\circ}$ ano dos anos finais, são as séries que apresentam uma quantidade menor de OAM.

Ilustrando com gráfico 1, temos o número de OAM por ano de ensino relacionados às habilidades propostos pela BNCC para cada ano de ensino. 
VII Congresso Brasileiro de Informática na Educação (CBIE 2018)

Anais dos Workshops do VII Congresso Brasileiro de Informática na Educação (WCBIE 2018)

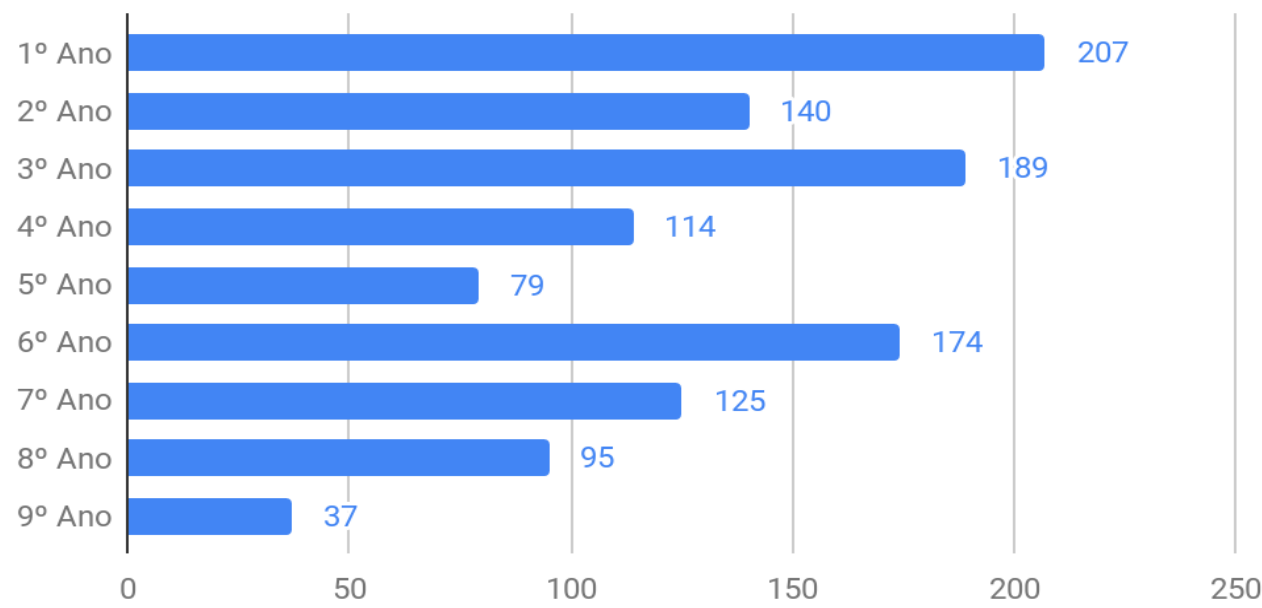

Gráfico 1. Quantitativo de apps catalogados por série no Ensino Fundamental.

Observa-se que a compreensão de relacionar termos como "Resolver e elaborar problemas cuja conversão em sentença matemática seja uma igualdade com uma operação em que um dos termos é desconhecido" ( $11^{\circ}$ habilidade da unidade temática Álgebra do $5^{\circ}$ ano) com o cotidiano do aluno, proporciona um caráter de uma disciplina "difícil" à medida que sentenças matemáticas com termos desconhecidos e fórmulas começam a surgir. Essa relação com realidade se torna cada vez mais abstrata, o que intimida estudante que não sabe onde usar tais habilidades nas suas práticas diárias.

Classificamos OAM que pertencem a mais de uma habilidade por ano de ensino, o que implica em um número maior de habilidades por objetos, ao se comparar com o número de apps analisados. Encontramos objetos que não se enquadram em nenhuma das habilidades, contabilizando 42 apps. Além desses, tivemos também o total de 37 OAM que foram descontinuados e não foram analisados por essa razão.

Para representar a categoria de app que não se aplica a habilidades trazidas pela BNCC do Ensino Fundamental, podemos citar o aplicativo Fit In The Hole (Figura 2), encontrado na categoria de jogos da Google Play Store, dentro da subcategoria puzzle. Para os níveis de ensino dos anos iniciais e finais, na unidade temática de Geometria, e nos descritores de habilidades para os anos iniciais, a respeito da classificação de habilidades, o app não se aplicou. Ambos os catalogadores, ao cruzarem as características matemáticas presente nas atividades do app com as habilidades dispostas na BNCC, sendo representado pelo descritor EF00MA00, não chegaram a um descritor de habilidade comum.

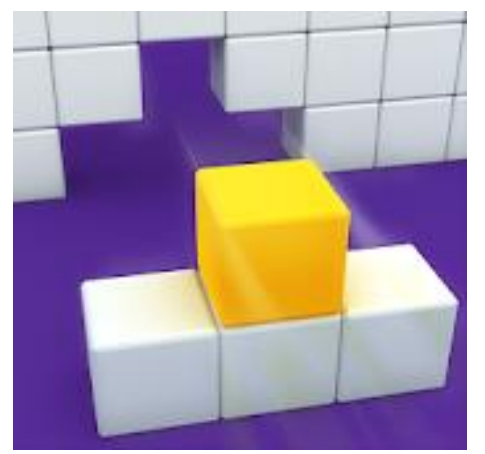

Figura 2. Ilustração de atividade do app Fit In The Hole.

Entre os apps classificados para as cinco unidades temática da BNCC, temos a unidade temática que se refere aos Números apresenta o maior percentual de apps 
classificados, com o total de 87 apps, seguida por Geometria com 48, Álgebra possui 31 e, com uma menor ênfase nas atividades dos apps, a Probabilidade e Estatística com 24 e Grandezas e Medidas com 18 apps. Isso reforça uma priorização por parte dos desenvolvedores os temas que trabalham com Álgebra, Números e Geometria, unidades temáticas que utilizam algoritmos básicos para a resolução exercícios e o raciocínio lógicomatemático e utilização de calculadoras, como já trouxeram Melo, Costa e Maia (2017) em seus estudos e levantamentos preliminares.

Observamos também, como consequência, a defasagem de apps produzidos para os temas de Grandezas e Medidas e Probabilidade e Estatística, unidades que cobram a capacidade de analisar dos dados fornecidos antes de qualquer cálculo matemático, exigindo do aluno uma leitura gráfica e conhecimento de unidade de medida que na grande maioria das vezes não explorado.

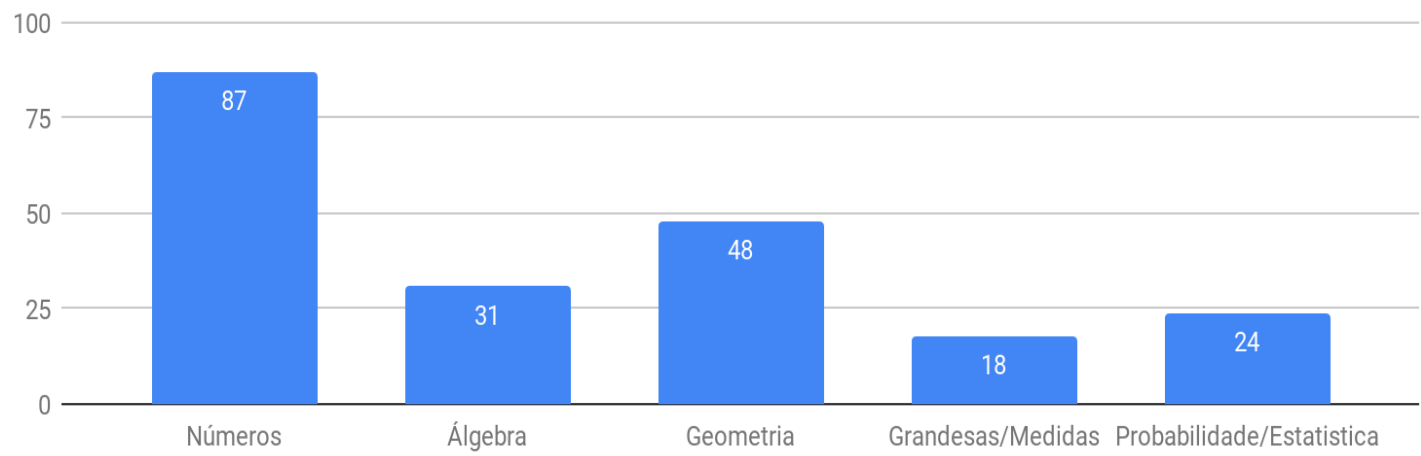

Gráfico 2. Quantidade de apps distribuídos por unidade Temática.

Mesmo sendo a Google Play Store, possivelmente, o repositório de apps mais popular para dispositivos móveis Android, o fato de contemplar todas as classes de apps pode dificultar a localização daqueles com potencial educativo por professores. Por esta razão, justificamos a criação de uma plataforma específica com apps educativos para Matemática por congregar os recursos em uma único lugar, pensada para facilitar o trabalho docente de seleção e avaliação do app, de acordo com sua aula, classificados em níveis de ensino, temas curriculares e descritores de habilidades de matrizes como a Prova Brasil e BNCC.

A seguir, apresentamos as considerações deste trabalho, a partir das inferências realizadas sobre os dados analisados.

\section{Considerações}

De maneira geral a nossa pesquisa traz um campo de estudo e inovação para o ensino de Matemática que precisa ser investigado e explorado. A popularização da informação de forma instantânea e dos dispositivos móveis demanda aos professores da atualidade a conhecer melhor seus alunos e as possibilidades pedagógicas que podem se servir. Os apps estão amparados por perspectiva pedagógica que provoca a aprendizagem dos conceitos Matemáticos. A catalogação desses recursos contribui para a prática docente na medida em que facilitará seu trabalho ao planejar e executar uma aula com suporte das TDIC.

A formação docente é importante para a consolidação de alguns resultados vistos durante a pesquisa, assim como contribuiu para os professores se apropriarem das ferramentas disponibilizadas pela Plataforma OBAMA. 
Alertamos para o cuidado que se deve ter ao escolher um app acessado em repositórios genéricos. Como identificamos, alguns apps apesar de classificados como instigadores do saber matemático, de acordo com os parâmetros de avaliação e classificação, não se enquadraram para o trabalho em nenhum tema de conteúdo ou descritor de habilidade da BNCC. Considerando que professores fizessem o mesmo procedimento de busca que empreendemos, teriam o mesmo resultado: quantidade elevada de apps que não correspondem às expectativas do levantamento, que é o princípio pedagógico. Esta é uma das motivações para o desenvolvimento da Plataforma OBAMA, onde o professor saberá que os recursos educativos digitais disponibilizados já estarão previamente avaliados e classificados de acordo com parâmetros de pesquisa específicos para matemática.

Disponibilizaremos os apps encontrados e os metadados deste trabalho na Plataforma OBAMA. Pretendemos ainda seguir o projeto com formações docente para o uso de TDICs, disponibilizar planos de aula com os apps, assim como a classificação dos apps para o ensino Médio com base na BNCC, concomitantemente a um novo processo de curadoria dos apps.

\section{Referências}

Base Nacional Comum Curricular (BNCC). Disponível em: $<$ http://basenacionalcomum.mec.gov.br>. Acesso em: 22 ago. 2018.

Batista, S.D. et al (2017). Reconstrução de um repositório de objetos de aprendizagem para Matemática. In: Congresso sobre Tecnologias na Educação (Ctrl+E), 2017, Mamanguape/PB. Anais do Ctrl+E 2017.

Castro-Filho, J. A.; Maia, D. L.; Castro, J. B. de; Barreto, A. L. de O.; Freire, R. S. (2016). "Das tabuletas aos tablets: tecnologias e aprendizagem da Matemática". In: Castro-Filho, J. A. et al. (Orgs.). Matemática, Cultura e Tecnologia: perspectivas internacionais. Curitiba: CRV, p.13-34.

CGI.BR. (2017). "Pesquisa sobre o uso das tecnologias de informação e comunicação nas escolas brasileiras: TIC educação 2017'. São Paulo: Comitê Gestor da Internet no Brasil.

Lemos, C. (2010). Jogos móveis locativos, cibercultura, espaço urbano e mídia locativa. In: Revista USP, São Paulo, n.86, p. 54-65, 64 junho/agosto.

Melo, E.M. et al (2017). Problemas para a Inserção das Tecnologias Digitais de Comunicação e Informação nas Escolas Públicas da Grande Natal: Um Levantamento entre Professores de Matemática. In: III Workshop da Licenciatura em Computação (CBIE 2017 - WLIC), 2017, Recife.

Melo, E. M.; Costa, C. J. N.; Maia, D. L. (2017) . Recursos educativos digitais para Educação Matemática: um levantamento para dispositivos móveis. In: Congresso sobre Tecnologias na Educação (Ctrl+E), 2017, Mamanguape/PB. Anais do Ctrl+E 2017.

Muniz, M. I. P. A. (2015). Usabilidade pedagógica e design de interação: processos de comunicação e colaboração em ambientes virtuais de aprendizagem. Rio de Janeiro, 2015. Tese (Doutorado) - Centro de Teologia e Ciências Humanas, Pontifícia Universidade Católica do Rio de Janeiro.

OCDE. (2016). Alunos de baixo desempenho: por que ficam para trás e como ajudá-los? Paris: OECD Publishing.

Oliveira et al (2017). Reconstrução de um repositório de objetos de aprendizagem para matemática. In: Congresso sobre Tecnologias na Educação (Ctrl+E), 2017, Mamanguape/PB. Anais do Ctrl+E 2017. 
VII Congresso Brasileiro de Informática na Educação (CBIE 2018)

Anais dos Workshops do VII Congresso Brasileiro de Informática na Educação (WCBIE 2018)

QEDU (2018) Comparando o aprendizado: Rio Grande do Norte. Disponível em: http://www.qedu.org.br/estado/120-rio-grande-do-norte/compare.

Saccol A., Schlemmer, E.; Barbosa, J. (2011). m-learming e u-learning - novas perspectivas da aprendizagem móvel e ubíqua. São Paulo: Pearson.

Wiley, D. A. (2008). "Connecting learning objects to instructional design theory: a definition, a metaphor, and a taxonomy”.Logan: Utah State University. 